MUZIKOLOSKI ZBORNIK - MUSICOLOGICAL ANNUAL XVII/1, LJUBLJANA 1981

UDK 778.62

\title{
ÜBER NEUE HANDSCHRIFTLICH ÜBERLIEFERTE LAUTENTABULATUREN
}

\author{
Wolfgang B o etticher (Göttingen)
}

Das Korpus der handschriftlich überlieferten Tabulaturen für Zupfinstrumente (Laute, Gitarre und deren Abkömmlinge, auch vereinzelt zum Gebrauch von Streichinstrumenten) ist jüngst in einem Katalog, 726 Objekte in 204 Bibliotheken erfassend, beschrieben worden, ${ }^{1}$ wobei ältere Versuche einer geschlossenen Darstellung, zuletzt vom Verfasser, ${ }^{2}$ ganz erheblich erweitert werden konnten. War damit eine $\mathrm{Mu}-$ sterung des Gesamtvorrats durchgeführt, so verbleibt doch als wichtigstes Desiderat der Forschung eine »inhaltliche Bestimmung " dieses Repertoires, das zunächst nur in einer äußeren Deskription, allerdings bereits mit vielen Hinweisen auf die Schreiber und die Provenienz der Handschrift, zur Darstellung gekommen ist. Über diese dringend erforderliche Katalogisierung sämtlicher Objekte nach den dort verzeichneten Satztiteln und Komponisten- bzw. Intavolatornamen hat der Verfasser an anderem Ort ${ }^{3}$ Überlegungen angestellt: immerhin handelt es sich um einen Totalvorrat von 22.000 Sätzen, die zu ca. $70 \%$ anonym verzeichnet sind. Satztitel fehlen bei ca. $40 \%$. Eine Konkordanzbestimmung, auch eine Identifikation der Vokalvorlage einschließend, ist bei dem augenblicklichen Stand der Forschung nur bei eng zusammengehörigen Handschriftengruppen möglich und wird auch bereits in vielen

1 Verfasser, Répertoire International des Sources Musicales (RISM), Reihe B, vol. VII, München 1978, 374 Seiten.

2 Verfasser in: MGG, Artikel Laute, VIII (1960), S. 356 ff. und Artikel Gitarre, V (1956), S. 108 ff. Vorangegangen war eine Übersicht der Quellen im Register seiner Habilitationsschrift Studien zur solistischen Lautenpraxis des 16. und 17. Jahrhunderts, Berlin 1943, S. 320-395, das französisch übersetzt Paris 1956 erschien (Bibliographie des sources de la musique pour luth, ed. C. N. R. S., S. 1-69).

3 Verfasser, Zur inhaltlichen Bestimmung des für Laute intavolierten Handschriftenbestands, in: Acta musicologica LI (1979), fasc. II, S. 193-203, ferner derselbe in: Festschrift K. G. Fellerer zum 70. Geburststag, Köln 1973, S. 50 ff. (Über Stand und Aufgaben der Erforschung der Tabulaturen für Zupf- und Streichinstrumente). 
Ansätzen geboten. ${ }^{4}$ Ferner liegen ergänzend dankenswerte Monographien zu einzelnen Tanz- und Liedtypen vor, die sich auf bestimmte Handschriftengruppen der Tabulaturart beziehen. ${ }^{5}$ Eine totale Inventarisierung aller Incipits in einem thematischen Katalog bleibt bei der Materialfülle undurchführbar und wäre auch lexikographisch wenig sinnvoll. Stattdessen ist ein abgekürztes, approximatives Verfahren die einzige gangbare Lösung, um dieses Repertoire, das in hoher Dispersion in 24 Ländern vorliegt, zu eröffnen und in seiner Gesamtheit vor Augen zu halten. Der Verfasser hat ein solches "dépouillement sommaire" genauer begründet. ${ }^{6}$ Er wird ein derartiges Titelverzeichnis der Sätze und der Nomina der Komponisten, also ein Totalregister der beiden wichtigsten und unverzichtbaren Daten einer Handschrift, in Kürze vorlegen. ${ }^{7}$ Damit werden besonders die selteneren Satztypen und die nur vereinzelt auftretenden Nomina in Erfahrung gebracht. Die Dringlichkeit eines solchen dépouillement sommaire ist doppelt evident, wenn man bedenkt, daß über das parallele Handschriftenkorpus der Orgeltabulaturen noch nicht annähernd ein Überblick geboten ist.

Erscheint mithin die inhaltliche Bestimmung in Bälde gesichert, so bleibt das Auge des Forschers weiter auf jene Objekte gerichtet, die bisher nur unzureichend bekannt oder gänzlich verborgen geblieben sind. Da keine Bibliographie ans Ende kommt, hat der Verfasser 1979 einen ersten Nachtragsbericht vorgelegt, ${ }^{8}$ dem jüngst noch eine weitere Untersuchung gefolgt ist, die sich der ältesten Überlieferung (vor 1530) und ihren - z. T. unbekannten - Quellen zuwendet. ${ }^{9}$ In dem vorliegenden Beitrag für den Jubilar, dem der Verfasser die nähere Kenntnis der Lautentabulatur von Škofja Loka verdankt, ${ }^{10}$ soll nun

4 zum Beispiel: J. Ward, The lute books of Trinity College Dublin, in: The Lute Society Journal IX, London 1967, S. 17 ff. und X, London 1968, S. $15 \mathrm{ff}$.

5 zum Beispiel: W. Kirkendale, L'Aria di Fiorenza id est Il Ballo del Gran Duca, Florenz 1972; J. Ward, The 'Dolful Domps', in: Journal of the American Musicological Society IV (1951), S. $111 \mathrm{f}$.

6 Acta Musicologica a. a. O., vorausgegangen waren im Prinzip gleichlaufende Erwägungen von J. Jacquot in: Le luth et sa musique, vers une organisation internationale des recherches, ibid. XXX (1958), S. $89 \mathrm{ff}$.

7 in der Reihe: Quellenkataloge der Musikgeschichte, Wilhelmshaven. Dort ist auch ein Generalregister geführt, das - alle Schreibvarianten einschließend - sämtliche Angaben der »äußeren Deskription « (RISM B VII) erfaßst. Für RISM B VII hat jüngst in dankenswerter Weise Chr. Meyer-Strasbourg ein »Register der Tabulaturgattungen und Namen«, München s. a. (1979) nachgeliefert (S. 3-12), das eine erste Orientierung gewährt.

8 in: Acta musicologica, a. a O,. S. $198 \mathrm{ff}$

- Verfasser, Zum Problem der ältesten handschriftlich überlieferten Lautentabulaturen, in: Ars Musica, Musica Scientia, Festschrift H. Hüschen, Köln 1980, S. 61-65.

10 RISIM a. a. O., S. 322, vgl. bereits D. Cvetko, Zgodovina glasbe na Slovenskem I, Ljubljana 1958, S. 268 und J. Mantuani. Pasijonska procesija $v$ Loki, Ljubljana 1917 (sowie in: Carniola VII, 3 und VIII, 1-2, Ljubljana 1916, 1917). 
eine erste Zusammenschau aller seit RISM VII hinzugewonnenen Objekte geboten werden, verbunden mit einer vorläufigen inhaltlichen Deskription. Bemerkt sei, daß bis jetzt es in keinem Fall gelungen ist, von den seit 1945 als "verschollen « in RISM bezeichneten Hss. (namentlich ehemals Berlin, Sorau, Danzig, Königsberg) etwas zurückzugewinnen: hier ist dasjenige, was der Verfasser noch aus seinen Aufzeichnungen 1936-1944 bewahrt hat, das einzige Zeugnis, sofern nicht glücklicherweise ältere Berichte erreichbar sind.11

Als neu seien hier - geordnet nach Ländern - die folgenden Handschriften aufgewiesen: Zunächst in der Bundesrepublik Deutschland ein im Privatbesitz befindlicher Kodex der »Domänen-Kanzlei des Fürsten Löwenstein-Wertheim-Rosenberg/Fürstenberg « in Wertheim am Main, Ms. Nr. 6, mit der sehr frühen Datierung $» 1525$ « bei einem Satz. Das Konvolut hält als Nomina Josquin, Isaac, Philomusus, Paul Hofhaimer und - möglicherweise der Schreiber - Adolf Blinhamer (auch: $A B$ ) fest. Hier ist ein gewichtiges Glied der frühen deutschen, wahrscheinlich Nürnberger Lautenpraxis gewonnen, das die Ebene um 1525 ergänzt, die jüngst auch in Wien einen Zugang mit einem Heftchen des Studenten Jacob Thuerner erfahren hat. ${ }^{12}$ Gemeinsam mit dem sog. »Königsteiner Liederbuch «, einer noch primitiven einstimmigen Aufzeichnung, ${ }^{13}$ ist damit die untere Grenze deutscher Lautentabulatur ganz neu gezogen worden. Wenig später informiert über weitere 7 deutsche Liedsätze (neben einigen französischen, lateinischen, englischen und italienischen Sätzen) die $\gg 1556$ « datierte Tabulatur des Matthias Greck, die sich in der Privatsammlung Schermar, lange im Nordturm des Ulmer Münsters aufbewahrt, erhalten hat. ${ }^{14}$ Hier ist wie an Konkordanzen zu erkennen - ein Repertoire in Nachbarschaft $\mathrm{H}$. Gerles und $\mathrm{H}$. Newsidlers geboten; von beiden Lautenisten sind dort aus dem Nachlaß des Marcus (bzw. Antonius) Schermar auch Tabulaturdrucke erhalten. - Auch die jüngere Spielpraxis wurde mit Funden in der BRD bereichert, ergänzend RISM. Nur im Umriß ${ }^{15}$ sei angezeigt ein merkwürdig verborgen gebliebener Kodex am Wirkungsort des Ver-

11 zum Beispiel O. Gombosi, Eine deutsche Lautentabulatur, in: Ungarische Jahrbücher III, Berlin 1923, S. $401 \mathrm{ff}$. (die verlorenen Berliner Bestände betreffend). Zahlreiche verschollene Objekte hat der Verfasser noch in seiner Habilitationschrift (Berlin 1943) mit Notenbeispielen festgehalten.

12 Der Verfasser ermittelte 1964 die - bei Mantuani nicht näher bezeichnete - Handschrift in Wien, Österr. National-Bibliothek, Handschriftenabteilung, Ms. 9704 und führt sie bereits in RISM, S. 358. Neuerdings auch Flotzinger in: Musik Alter Meister XXVII, Graz 1971, S. V ff.

13 Berlin-West, Staatsbibliothek, Handschriftenabteilung, Ms. germ.qu. 715, vgl. RISM, S. 17 f., die wohl älteste Aufzeichnung in deutscher Lautentabulatur (möglicherweise auch für Streichinstrument). Merkwürdigerweise sind die musikalischen Beigaben der seit langem der Germanistik vertrauten Handschrift bis jüngst übersehen worden.

14 Jüngst überführt nach Ulm, Stadtbibliothek, Ms. $131 \mathrm{~b}$.

15 es sei auf ergänzende Angaben in Acta musicologica, a. a. O. verwiesen: S. $199 \mathrm{ff}$. 
fassers, in Göttingen, ${ }^{16}$ um 1740 (mit Logy bzw. Comte, Meley, Heins., Weise bzw. Weiß), eine Gruppe von fünf ebenfalls in französischer Lautentabulatur aufgezeichneten Hss. in Freising ${ }^{17}$ um 1790-1800, ferner eine Hs. aus dem Nachlaß des Grafen von Toerring-Jettenbach in München ${ }^{18}$ um 1755-1765, endlich weitere Objekte der genannten Ulmer Sammlung, ${ }^{19}$ die aber wesentlich jünger als das Ms. Greck sind. In Stuttgart ${ }^{20}$ enthält ein handschriftlicher Anhang an den Druck des J. F. B. C. Majer, Museum Musicum (Schwäb. Hall 1732) eine italienische Tabulatur für Cyther, um 1641, die neben deutschen Liedsätzen einen wichtigen Traktat zur »Applicatio " und den »Manieren " etc. aufweist. Während in der Deutschen Demokratischen Republik nur ein kleiner Fund in Dresden ${ }^{21}$ aus der ehemaligen Privatsammlung des sächsischen Königs nachzutragen ist (1 Blatt für Gallichon, zu der in RISM, S. 88 bis 92 beschriebenen Gruppe gehörig), ist der Zuwachs an neuen Quellen in Österreich erheblich. Jüngst ist aus dem Nachlaß der Familie des Grafen Harrach zu Wien ${ }^{22}$ ein umfänglicher brauner Lederband mit 65 beschriebenen Bll. aufgetaucht, mit Sätzen von Lauffensteiner, Riechtaller (Hirschthaler), Meuhel (Meusel) und Melay und einem tombeau auf den Tod des Kaisers Joseph I. (1710), mit vielen Suitensätzen, auch piano-forte-Effekten, um 1755-1760. Zwei kleine Faszikel am gleichen Ort'23 treten hinzu: Mss. 10.065/127 (1 Doppelblatt) um 1720 und XIV 3102/Sch (2 Blätter), beide für Mandora. Eine sehr fragmentarische Tabulatur hat sich in $\mathrm{Melk}^{24}$ angefunden, es sind Reste eines früher viel größeren Bestands, Ensemble-Sätze mit einer intavolierten Stimme und defekten übrigen Stimmen en musique.

16 Göttingen, Niedersächsische Staats- und Universitäts-Bibliothek, Handschriftenabteilung, Ms. $8^{0}$ Philos. $84 \mathrm{k}$.

17 Freising, Dombibliothek. Bis 1970 aufbewahrt in Weyarn (Bayern), Musikarchiv des Augustinerchorherrenstifts. Mss. Wey 662, 663, 664, 682 und 692. Im wesentlichen ist dieses Repertoire verbunden mit Joseph Michael Zinck (1759-1829). Es dürfte sich um eine der spätesten Tabulaturengruppe schlechthin handeln.

18 Jüngst München, Bayerische Staatsbibliothek, Musiksammlung, Acquisitions-Nummer G. 74/352.

19 Ulm, Stadtbibliothek, Mss., $133 \mathrm{a}, 132,132 \mathrm{~b}, 133 \mathrm{~b}, 239$, alle um 1620-1630, Datierungen 1626, mit überwiegend französischem Repertoire, doch sind auch $S$. Scheidt und V. Haußmann genannt. Primär für Mandora.

20 Stuttgart, Württembergische Landesbibliothek, Signatur R. 18. Maj. 1. Es ist das Handexemplar Majers, sehr wahrscheinlich stammen auch die Eintragungen von ihm.

21 Dresden, Sächsische Landesbibliothek, Ms. 3065/V/3, alte Signatur: CII a, jüngere Signatur: 29/V/1.

${ }_{22} \mathrm{Im}$ Depositum Harrach in Wien, Allgemeines Verwaltungsarchiv des Österr. Staatsarchivs. Ich verdanke den Hinweis Herrn Dr. Biba von der Bibl. der Gesellschaft der Musikfreunde, Wien. Jüngst auch ein verdienstlicher Index von J. Klima (Wiener Lautenarchiv Nr. 9, 1976). Wichtige Teile der Harrach-Sammlung befinden sich in New York (RISIM, S. 237 f.).

23 Wien, Bibliothek der Gesellschaft der Musikfreunde.

24 Melk, Benediktinerstift, Ms. VI, 1840, Nr. 1; Ms. VI, 1987, Nr. 7; Ms. VI, 1836, Nr. 28. Jüngst vgl. J. Klima, Wiener Lautenarchiv Nr. 19, 1978. 
Auch in dem sonst vorzüglich überschaubaren Tabulaturenbestand von Kremsmünster ${ }^{25}$ sind noch solche Reste zu erwarten. Zwei bedeutende Funde treten aber hinzu, die in RISM ebenfalls fehlen: zunächst in Linz, aber an einem dort nicht vermuteten Ort, ${ }^{26}$ ehemals Archiv Aurolzmünster, Ms. 465. Hier ist wieder eine späte Quelle verfügbar, mit italienischen und lateinischen Liedsätzen, Motettenumschriften. Genannt sind H. L. Hasler, A. und G. Gabrieli, L. Marenzio, Cl. Merulo, H. Vecchi, neben J. Berentius, W. Dachstein, G. Eremita, B. Gesius, K. Herbert, P. Speratus, ferner 9 Duos eines Giusoffo Biffi (über deren Provenienz, vgl. zu Biffi Eitner, Quellenlexikon II, S. 40, noch eine Bestimmung ansteht). Schreiber war ein (wohl aus Nürnberg zugereister) Michael Eysert, der umfassende Bildung und Repertoirekenntnis (auch englischer Vorlagen) verrät, immerhin sehen wir ca. 250 Sätze auf 95 Blättern. Ein ungewöhnlicher Fund ergab sich jüngst in der Privatbibliothek des Grafen von Goess in Schloß Ebenthal bei Klagenfurt (Kärnten). Der Verfasser hatte bei mehrtägigem Aufenthalt Gelegenheit, den Gesamtbestand in Autopsie (also nicht nur über Mikrofilmierung) zu erforschen, nach den dankenswerten Hinweisen von Prof. Federhofer, der als ehemaliger Grazer Musikwissenschaftler als erster auf diese Funde aufmerksam machte, und nach dem Vorgange der Bemühungen von Dr. J. Klima-Wien und Dr. D. A. Smith (USA) in den letzten Jahren. Es handelt sich um 13 Volumina aus dem Nachlaß des Johann Peter v. Goess (1667-1716), der als Knabe von Holland nach Österreich kam, und in nächster Generation von Maria Anna v. Thürheim und Jacobina v. Thürheim, in weiterer Generation von Maximiliana v. Goess und deren Lautenlehrer, einem Anton Joseph Hueber (um 1740). Die meisten Tabulaturen (überwiegend für Laute, einige für Viola, Theorbe) sind jedoch älter und zeigen ein sehr interessantes, noch nicht verwertetes Repertoire französischer Provenienz (du Faut, Vieux Gaultier, Gaultier, du But, de Fresneau, Bouquet, Mercure, Hotman, Mouton, Dupré, Pinel), englischer Provenienz (William Lawes, John Jenkins, Charles Coleman, Steffkins, W. Young, Ives, Quilekorn, Poll), ferner die im deutschen Sprachbereich mehrmals in Tabulaturen belegten Herbich, Conte de Logy, Franz Ginter, Pater Swironi, Wolff, Bartolomi, St. Luc, Reusner, Betkoffsky, Angelo Michielo. Im ganzen sind es über 850 Sätze, die zu ca. $40 \%$ bezeichnet und mit Komponistennamen versehen sind. Mithin eine erhebliche Bereicherung des bisher bekannten Repertoires 1660-1740, wobei die Einheitlichkeit der Überlieferung aus Besitz einer einzigen Familie schwerwiegt. Das Dépouillement wird der Verfasser im Rahmen der angekündigten Gesamtdarstellung der nunmehr ca. 785 Handschriften (»dépouillement sommaire«) in

25 vgl. RISM, S. 155-161 und die dort genannte Literatur.

${ }^{26}$ Linz, Oberösterr. Landesarchiv. Der Verfasser hatte bereits in RISM, S. 171, eine andere Tabulatur in der Bundesstaatlichen Studienbibliothek nachgewiesen. Vgl. jüngst J. Klima, Wiener Lautenarchiv Nr. 18, 1977. 
Kürze in Druck geben. Immerhin bedeuten die jüngsten Zugänge aus Österreich mit Ms. Linz (um 1600) und den späteren Mss. Ebenthal eine beträchtliche Vertiefung unserer Kenntnis. Es kann hier nur ein vorläufiger Bericht der intensiven Konkordanzstudien geboten werden, deren Resultat an anderem Ort (Anmerkung 7) systematisch vorgelegt wird, was eine wichtige Lücke schließen soll.

Nur am Rande seien Einzelobjekte, die ebendort zu ergänzen sind, aufgeführt. Und zwar in Budapest, ${ }^{27}$ London, ${ }^{28}$ Bologna, ${ }^{29}$ Florenz, ${ }^{30}$ Utrecht $^{31}$ und Brüssel. ${ }^{32}$ Auch Modena, ${ }^{33}$ sowohl im Archivio di stato als in der Estensischen Sammlung bereits nachgewiesen (RISM, S. 209-213), ergänzt sich mit 4 weiteren Handschriften. Kleinere Funde in Polen ${ }^{34}$ seien nur gestreift, um vollständig zu berichten. Ein entscheidender Fund aber kam dem Verfasser nach einer kürzlichen Studien- und Vortragsreise in Japan hier zum ersten Male in die Hände: ${ }^{35}$ das seit langem gesuchte "Livre de luth" aus der ehemaligen Sammlung Dr. W. H. Cummings-London, die auch für Orgeltabulaturen bekanntlich höchsten Wert besitzt. Das Ms. (alte Signatur 91), mit Exlibris WHC (Nr. 247) enthält auf Fol. 1-2, 3v-6r, $7-10,11 \mathrm{v}-17,18 \mathrm{v}-20,30 \mathrm{v}-31$ (31 Bll. im ganzen) französische Lau-

27 Budapest, Ungarische Akademie der Wissenschaften, Handschriftenabteilung, Ms. K. 53/II (um 1564), ferner ebendort, Nationalbibliothek Széchényi, eine Einzeichnung in den Druck M. Waissel, Tabulatura, Frankfurt/ O. 1573, Sammlung Bártfa (Bartfeld), um 1585.

28 London, Lambeth Palace, Ms. 1041 (1620-1640).

29 Bologna, Biblioteca. Universitaria, Ms. 596. H. H. 2. 4 (um 1500), eine wohl früheste Aufzeichnung in italienischer Lautentabulatur.

30 Florenz, Bibl. Nat. Centr. Mss. Magl. VII, 618; VII, 1222 bis (beide 1640-1660), VII, 894 (1633-1640). Zu übrigen Mss. an diesem Fundort vgl. RISM, S. $107-117$.

31 Utrecht, Universitätsbibliothek Hs. Anhang in Druck Phalèse. Ferner in Utrecht-Zeist, Bibl. der Herrenhuter-Gemeine, eine Hs.

32 Brüssel, Bibl. du Conservatoire Royal, Ms. 24.135. Weitere IMss. an diesem Fundort vgl. RISM, S. 53-57.

${ }_{33}$ Modena, Bibl. Estense, Mss. Mus. E. 323, F. 1528, G. 239, G. 289/2.

34 Wrocław (ehem. Breslau), Bibl. Uniwersytecka, hs. Anhang an den Druck S. Kargel, Lautenbuch..., Straßburg 1586. Signatur: Muz. 50075. Um 1590, 1 deutscher Liedsatz. Ferner Warszawa, Bibl. Narodowa, ehem. Bibl. Załuski, Ms. Mus. 2088, eine Gitarrentabulatur um 1765 mit ca. 40 Sätzen verschiedener (auch französischer) Provenienz. Jüngst liegt ein vortrefflicher Bericht über die Lautentabulatur der Bibliothek H. Eopacinskiego in Lublin (VR Polen) Signatur 1985 von Zofia Stęszewska vor: in Muzyka, Kwartalnik Póswięcony, historii i teorii muzyki, Warschau (Polska Akademia Nauk) XXV, Nr. 3, 1980 S. 85-114.

35 Der Verfasser dankt Frau Kollegin Professor Noriko Takano von dem Music Research Institute des Kunitachi College of Music in Tokyo und gleichermaßen Herrn Kollegen Professor Ko Tanimura von der Universität Osaka, Faculty of Letters, Abteilung Musikwissenschaft, für liebenswürdige Unterstützung bei der Erforschung der jetzt in Tokyo, 7-6-41, Akasaka-cho, Minato-ku, befindlichen Nanki Music Library. Im übrigen sei auf den kursorischen Katalog (Tokyo 1970, S. 9) verwiesen. Das Objekt hat jetzt die Signatur: N-4-42. 
tentabulatur (auch für Theorbe, z.T. bis Ziffer »13《Baßsaiten führend). Neben Anweisungen (»how to play the lute by eights in its own tuning «), auch Übertragungen von Generalbaßakkorden haben die (mindestens 3) Schreiber, offenbar Engländer, ${ }^{36}$ einheimische und italienische Liedersätze (auch begleitend) festgehalten, ferner aus französischen Quellen z. B.: »Old Gautiers Nightingall « neben 2 Sätzen »Menuett«. Daß auch eine italienische Vorlage bekannt war, besagt die Notiz: »set vpon the lute By C. Morellj«. Für Didaktik des Lautespiels zeugen Übungsstücke: $» 8$ times, after This Leaf is played begin the first Line eleven times". Auch eine "Toccata « dient dem Übungszweck. Das Ms. entstand Ende des 17. Jahrhunderts (Wasserzeichen: Doppeladler). Die Odyssee der Nanki-Bibliothek, 1935 in Besitz von Kyubei Ohki gelangt, führte über verschiedene externe Aufbewahrungsorte (wo leider auch Verluste eintraten) und hat schon das Interesse von Thurston Dart und seinem Cambridger Schüler Hugh McLean 1960 erregt, Januar 1967 bemühte sich J. H. Davies, Musikbibliothekar des $B B C$ in London, erneut um eine Bestandsaufnahme dieser kostbaren Kollektion. Unser Objekt scheint Cummings von einem französischen Antiquar erworben zu haben (Ausschnitt aus einem Versteigerungskatalog eingeklebt). Eine exakte Würdigung mit Konkordanz wird der Verfasser an anderem Ort (vgl. Anm. 7) vorlegen, verbunden mit der äußeren Deskription. Gegenüber RISM, S. 331 bedarf es bei einer anderen japanischen Handschrift eines Nachtrags: Das dort verzeichnete Ms. »ohne Signatur (I)« ist jetzt als Ms. Mus. 211 in der Musashino Academy of Music (= Ongaku Daigaku), Tokyo, geführt. Das Objekt, bisher nur unzulänglich bekannt,,37 war dem Verfasser für RISM nicht in Autopsie erreichbar gewesen. Nunmehr ergab eine Prüfung, daß hier ein wichtiger Zugang zur französischen Theorbenpraxis (14 chörig) geboten ist. Namentlich Robert de Visée (hier: Vizee) erscheint in diesem Repertoire. ${ }^{38}$ Der französische Schreiber ${ }^{39}$ hat wichtige Spielanweisungen, auch spezielle Variationentechniken (double, en redouble, autrement) demonstriert und damit die spärliche Theorbenliteratur bereichert. Auch hier darf auf die interne Würdigung im Rahmen des erwähnten dépouillement sommaire verwiesen werden. Mit diesem Neuzugang von 57 Objekten, immerhin $6 \%$ des RISM-Bestands, ist im

36 allerdings mit schwankender Orthographie, z. B. tuning und tooning; man bevorzugt beim Umblättern das italienische torna.

37 Versteigerungs-Katalog H. Schneider Nr. 76, Tutzing 1955, S. $40 \mathrm{f}$. (als Nr. 343) mit 1 Faksimile S. 91. Der auftretende Musikername »otoman« verweist nicht, wie hier vermutet, auf einen »türkischen « Lautenisten, es ist der französische Gambist Hotman (Hottemann) gemeint, der in verschiedenen intavolierten Quellen belegt ist.

38 Unter den handschriftlichen Quellen zu ergänzen im Artikel Visée, MGG XIII (1966), S. 1832 (F. Lesure).

39 Merkwürdig verderbt der berühmte Titel Lin mortele (= L'Immortelle), der aber auch danach in korrekter Rechtschreibung erscheint (Gaultier). 
Zeitraum von drei Jahren der Überblick nicht unerheblich erweitert worden, wobei eine breite Streuung von sehr frühen, mittleren und sehr späten Handschriften die Kenntnis des Repertoires vertieft.

\section{POVZETEK}

Avtor, ki je izdelal »Opisni katalog tabulatur za lutnjo in kitaro v rokopisu" (Beschreibender Katalog der Lauten- und Gitarrentabulaturen in Manuskript«, RISM, Reihe B, Volumen VII, 1979), razpravlja o možnosti in nujnosti, da se izvede vsebinska opredelitev repertoarja okrog 22.000 skladb. Izhajajoč iz svojih zadnjih razlag v Acta musicologica (1979) daje napotke, kako bi se dalo celoten inventar imen skladateljev in oznak skladb sistematično leksikografsko prikazati po "skrajšanem" postopku. Takšen dépouillement sommaire, izdelan ob sodelovanju francoskih raziskovalcev, naj bi v kratkem omogočil poznavalcu dostop do repertoarja, ki ga je bilo doslej mogoče preučevati le $\mathrm{v}$ ozkih odsekih. Razen tega opisuje avtor nove najdbe rokopisov $\mathrm{z}$ omenjenim načinom zapisovanja, $\mathrm{ki}$ jih mora dodati po izidu njegovega kataloga. Vsega skupaj je naknadno še kar 50 primerov, pri čemer izstopa eno najdišče z nič manj kot 13 tabulaturami (približno 850 skladb). Neodvisno od omenjenega dépouillementa najavlja avtor še suplement za RISM in podaja pregled njegove vsebine. 